\title{
The effect of simultaneous and successive presentation of information processing training upon concept learning
}

\author{
J. L. DUNHAM, DANIEL D. BLAINE, AND MARK B. REEVE, \\ DEPARTMENT OF EDUCATIONAL PSYCHOLOGY, THE \\ UNIVERSITY OF TEXAS AT AUSTIN, Austin, Tex. 78712
}

The purpose of this study was to examine the relationship of associative memory $(A M)$ to information processing training (IPT) under minimum and maximum memory requirements and to determine the effect of both $A M$ and IPT upon subsequent performance in a concept learning task (CT). Superior performance in IPT was observed with a decrease in memory requirement. Successful performance in the IPT phase improved performance on the $C T$, regardless of the memory requirement during IPT or the level of $A M$

Cahill \& Hovland (1960), Kates \& Yudin (1964), and Miller \& Davis (1967) have demonstrated that superior performance in concept learning is attained when the concept instances are presented simultaneously rather than successively. Other investigations (e.g., Bourne, Goldstein, \& Link, 1964; Pishkin, 1967; and Pishkin \& Wolfgang, 1965) have found that intermediate levels of available past instances also have a facilitative effect on concept learning performance. This facilitative effect has typically been interpreted as a reduction in the load placed on S's memory. There is little evidence, however, to indicate how this reduction specifically affects the solution processes utilized by Ss in concept attainment.

If $\mathrm{S}$ were to employ a strategy in which he tested hypotheses about relevant dimensions, then, by comparing two instances, he could gain information concerning the status of a hypothesis. For example, if two instances have different category designations but the dimensions involved in the hypothesis have the same values, the hypothesis would be rejected. Conversely, if the category designations are the same and the values are different, the hypothesis would again be rejected. These comparisons are highly dependent upon memory, i.e., recall of past instances is necessary to make these comparisons.

It seems apparent that formulating such a strategy would also be highly dependent upon the recall of past instances in the concept task. If, however, Ss were trained to process information about the status of a hypothesis when the necessity of recalling past instances was removed, Ss should be more successful in a subsequent concept attainment task. The major purpose of this study was to investigate the effect of information processing training (IPT) under simultaneous (SI) and successive (SU) presentation of instances upon subsequent performance in a concept learning task (CT). It was predicted that Ss who received the IPT under the SI condition would not only have more success in the IPT than Ss in the SU condition, but also would exhibit superior performance in the subsequent CT. That is, presenting instances simultaneously in the IPT should make it easier for Ss to see how decisions could be made about hypotheses in the CT.

Since the availability of previously presented instances was interpreted as reducing the load placed on S's memory, it was predicted that Ss low in associative memory would profit more from the SI presentation of IPT than Ss high in the ability. It was expected that the extent to which Ss were successful with the IPT would be indicative of success in CT performance. Consequently, it was predicted that Ss low in memory would profit more from SI training with respect to their performance in the CT.

Subjects. The Ss were 44 undergraduates from two introductory educational psychology classes at The University of Texas at Austin.

Materials and Procedure. Except for certain segments of the instructions, all materials were programmed for presentation on IBM 1050 computer terminals connected to an IBM 1440 system. The CT was a four-category, two-dimensional conjunctive problem. The stimuli consisted of the letters $A$, $B, C$, and D, which always appeared in alphabetical order but could be either upper or lower case. The stimuli were presented by the method of anticipation.
The Ss were first given a memory test consisting of 16 items. For each item, Ss were presented with four different $\mathrm{CT}$ instances, each followed by a different category number. The instances were presented successively, after which, one of the instances was repeated. Ss were then asked to recall the number which followed that instance. The repeated instance was balanced for position in the four nonresponse instances and for the number which was the correct response.

In the next phase, the IPT, 36 information processing problems were presented. For each problem, Ss were instructed to consider a pair of letters as a hypothesis and then were presented with five instances, each followed by its category number. After presentation of the instances, Ss were asked whether the pair of letters could be rejected as possible relevant dimensions. In one-half of the problems, sufficient information was presented to reject the hypothesis; in the other half, there was insufficient information to reject the hypothesis. The problems were presented either under SI or SU conditions. In the SU condition, the instances were presented one at a time and then were spaced behind a facesheet before the next instance was presented. In the SI condition, the same problems were presented one at a time; however, the instances remained visible until a response had been made to the rejection question. In both conditions, Ss were given an unlimited response interval.

After completion of the IPT, Ss began the CT. The instances were presented successively to all Ss. Ss were given an unlimited response interval and, after responding, were told whether their categorization was correct or incorrect and what the correct categorization for the instance was. This procedure continued until $S$ achieved a criterion of 12 consecutive correct responses or had responded to 96 instances.

Results and Discussion. The mean score on the computeradministered memory test was 10.07 with a standard deviation of 3.02. The split-half reliability coefficient, estimated by the Spearman-Brown formula, was .72 .

An analysis of covariance upon number of correct problems in IPT, with training condition in IPT as the independent variable and memory score as a covariable, indicated that, as predicted, Ss trained in the SI condition did significantly better than those in the $\mathrm{SU}$ condition $(\mathrm{F}=6.16 \mathrm{df}=1 / 41, \mathrm{p}<.05)$. There was no significant interaction between Training condition and Memory ( $F$ $=.17, \mathrm{df}=1 / 40, \mathrm{p}=.68$ ), and furthermore, the slope of the regression lines with respect to memory were not significantly different from zero $(F=1.45, \mathrm{df}=1 / 41, \mathrm{p}=.23)$. That is, memory was inconsequential to the prediction of success in IPT. Apparently, the type of associative memory measured by the test was not the type necessary for recalling information used to form a strategy of testing hypotheses in the IPT problems. However, it still appears that memory is important, since $S s$ in the SI condition, which presumably had less memory requirement than the SU condition, were more successful with the IPT problems.

The mean number of correct problems in IPT was 28.73. However, the distribution was bimodal. One group of Ss clustered about the chance level (Mean $=19.07$, Range: 13-22). The other group was successful at solving IPT problems $($ Mean $=33.23$ Range: 27-36). Therefore, for purposes of analysis of the CT data, the scores in IPT were dichotomized.

An analysis of covariance upon number of errors in CT was computed, with the dichotomized IPT scores and the training conditions as the independent variables and memory as the covariable. Those high in IPT made significantly fewer errors in the CT than those less successful on the IPT $(F=13.56$, df $=1 / 39$, $p<.01)$. This lends support to the hypothesis that success on the IPT was indicative of processing information in a manner which facilitated performance on the CT. That is, being able to successfully test hypotheses. as measured by IPT, was more important to success in the (T than either the condition of training or the level of memory.

There was no significant difference between SI and SU conditions $(F=.30, \mathrm{df}=1 / 39, \mathrm{p}=.60)$, indicating that removing the memory requirement during the IPT did not increase the likelihood of successful performance in the CT. However, it might 
be expected that Ss in the SU condition would profit from the greater similarity between the presentation of the information processing problems and the CT. Apparently, any increased likelihood of success in the SI condition was not sufficient to overcome whatever gain occurred due to similarity.

Neither the interaction between memory and training condition nor the interaction between memory and dichotomized IPT scores was significant $(F=2.75, \mathrm{df}=1 / 38, \mathrm{p}=.10 ; \mathrm{F}=.04, \mathrm{df}=1 / 38, \mathrm{p}$ $=.84)$. However, the correlation between memory and the number of correct items on the CT was .55 $(p<.05)$ for Ss trained in SU and only .04 for Ss trained in SI. This suggests that those trained in the SI condition employed a strategy minimizing memory requirements, while $\mathrm{Ss}$ in the $\mathrm{SU}$ condition had less opportunity to develop this sort of strategy and were forced to rely upon a method of making decisions about hypotheses which was more dependent upon recall of past instances.

In conclusion, Ss who were successful in testing hypotheses about relevant dimensions were more successful in the subsequent CT, regardless of the condition of training or the level of memory. Removing the memory requirement during the IPT did not facilitate performance in the CT. However, removing the memory requirement did appear to aid $S s$ in formulating a method of testing hypotheses in the CT which was less dependent upon memory.

\section{REFERENCES}

BOURNE, L. E., Jr., GOLDSTEIN, S., \& LINK, W. E. Concept learning as a function of availability of previously presented information. $J$. exp. Psychol., 1964, 67, 439-448.

CAHILL, H. E., \& HOVLAND, C. I. The role of memory in the acquisition of concepts. J. exp. Psychol, 1960, 59, 137-144.

KATES, S. L., \& YUDIN, L. Concept attainment and memory. J. educ. Psychol., 1964, 55, 103-109.

MILLER, G. W., \& DAVIS, J. K. Memory in concept identification as a function of concept complexity, method of presentation and recall of type. Paper presented at the meeting of the American Educational Research Association, New York, February, 1967.

PISHKIN, V. Supplementary report: Availability of feedback-corrected error instances in concept learning. J. exp. Psychol., 1967, 73, 318-319.

PISHKIN, V., \& WOLFGANG, A. Number and type of available instances in concept learning. J. exp. Psychol, 1965, 69, 5-8. 